\section{How do you feel?}

Although over 60 genes are known to cause hearing loss, very little is known about the genetics of touch. A few genes that cause complete insensitivity to pain have been identified, but whether or how much touch sensitivity is influenced by genetics is not well understood. Gary Lewin and colleagues report an analysis of touch phenotypes in cohorts of healthy twins and show that genetics do indeed influence touch sensation (PLOS Biol. 10, e1001318, 2012). They find that the heritability $\left(h^{2}\right)$ of tactile acuity is 0.27 and that the heritability for vibration detection threshold is $\mathbf{0 . 5 2}$. As they hypothesized, the authors find that touch acuity and sensitivity are correlated with hearing ability (as all are mechanosensory traits) in hearing-impaired individuals. The authors also analyzed touch phenotypes in individuals with Usher syndrome type 2 who have relatively mild hearing loss. They observed that individuals with recessive mutations in USH2A have slightly impaired touch acuity and sensitivity compared to individuals in whom the pathogenic mutations are unknown. These results suggest that the senses of touch and hearing have shared genetic mechanisms. The authors conclude that more genes that regulate both hearing and touch sensation should be identified.

\section{piRNA, epigenetics and memory formation}

Piwi-associated small RNAs (piRNAs) function in the germline to silence transposons by transcriptional and post-transcriptional mechanisms. Now, Eric Kandel, Thomas Tuschl and colleagues show that piRNAs are expressed in the central nervous system in the sea slug, Aplysia, where they regulate transcription of the plasticity-related gene CREB2 (Cell 149, 693-707, 2012). The authors initially identified neuronal piRNAs in a small RNA library, and they also identified piRNAPiwi protein complexes in neural cells. They used cultured neurons to show that piRNAs are regulated by the neuromodulator serotonin and that Piwi functions to enhance serotonin-dependent long-term synaptic facilitation, as measured electrophysiologically in co-cultured sensory and motor neurons. They found that Piwi and the piRNA piR-F specifically repress $C R E B 2$, and they show that CREB2 is regulated by serotonin-dependent promoter methylation. This work suggests a broader role for piRNA-mediated regulation of expression in somatic tissues beyond their role in the germline and provides insight into epigenetic mechanisms of memory formation.

\section{ITGA3 and epithelial integrity}

Giuseppina Spartà and colleagues identify mutations in the ITGA3 gene (encoding integrin $\alpha 3$ ) as the cause of a rare congenital disorder characterized by disrupted basement membrane structures in multiple epithelial organs (N. Engl. J. Med. 366, 1508-1514, 2012). The authors examined three unrelated subjects with overlapping clinical features that included nephrotic syndrome, interstitial lung disease and blistering skin lesions. Histological and immunofluorescence analyses of affected skin samples showed disruptions in the basement membrane

Written by Orli Bahcall, Pamela Feliciano, Emily Niemitz \& Kyle Vogan and a lack of integrin $\alpha 3$ protein. Consistent with these findings, candidate gene sequencing identified homozygous ITGA3 mutations in all three affected individuals. The clinical features of this syndrome overlap with the phenotype of Itga3 knockout mice, which die shortly after birth with multiple defects in kidney, lung and skin tissues. Mutations in the gene encoding integrin $\beta 4$ (ITGB4), which forms functional heterodimers with integrin $\alpha 3$, cause a similar skin blistering phenotype (Nat. Genet. 10, 229-234, 1995). These findings underscore the importance of integrins in maintaining epithelial integrity and show how histological findings can aid clinical diagnoses and help elucidate the genetic causes of rare congenital disorders.

KV

\section{Genetics of blond hair}

Sean Myles and colleagues report on the genetic basis of blond hair in Solomon Islanders, a population that shows both the darkest skin pigmentation outside of Africa and the highest prevalence of blond hair (Science 336, 554, 2012). The authors conducted a genome-wide association study for hair color on 43 blond and 42 dark-haired individuals from the Solomon Islands. They selected the $10 \%$ of extremes for hair pigmentation distribution by spectrometric measurements, while controlling for age, sex and geography. They identified a single associated locus on chromosome 9p23, which includes the candidate gene TYRP1, which has previously been associated with variation in skin and hair pigmentation across several species. They genotyped a missense variant that showed the most significant association in 918 individuals from Solomon Island and estimate that this variant accounts for $46.4 \%$ of the variance in hair color. The associated allele is present at a frequency of 0.26 in Solomon Islanders, whereas, outside Oceania, this allele is rare or absent. Ancestry analysis using a worldwide reference sample confirmed the separation of Solomon Islanders from neighboring populations. The lack of systematic ancestry differences between blond and dark-haired individuals in the study suggests that the higher prevalence of blond hair was not attributable to recent gene flow from European populations.

$O B$

\section{Epigenetic activation by long RNA}

Facioscapulohumeral muscular dystrophy (FSHD) is associated with contraction of D4Z4 repeats on chromosome 4 , loss of repressive polycomb-mediated chromatin modifications and derepression of nearby genes. Now, Davide Gabellini and colleagues identify a long RNA produced from the contracted D4Z4 locus and show that it has a role in epigenetic dysregulation in FSHD (Cell 149, 819-831, 2012). The authors show that a transcript called $D B E-T$ is produced from the D4Z4 repeat in primary muscle cells from individuals with FSHD but not from healthy controls. They used rodent cells containing a single human chromosome 4 to show that $D B E-T$ is localized within the nucleus at the D4Z4 locus and that it binds to the trithorax group protein Ash1L. They also showed that ASH1L protein localizes to the FSHD locus and is more abundant in primary muscle cells from individuals with FSHD compared to those from healthy controls. The authors suggest a model in which contraction of D4Z4 repeats causes loss of polycomb silencing and inappropriate production of $D B E-T$, thereby leading to recruitment of $\mathrm{ASH} 1 \mathrm{~L}$, chromatin remodeling and derepression of nearby genes in FSHD. 